\title{
Development of a powerful UCN source at PNPI's WWR-M reactor
}

\author{
Anatolii Serebrov ${ }^{1, \text { a }}$, Vitalii Liamkin ${ }^{1}$, Aleksey Fomin ${ }^{1}$, Valeriy Pusenkov ${ }^{1}$, Konstantin Keshishev ${ }^{2}$, Sergey Boldarev $^{2}$, \\ Dmitriy Prudnikov ${ }^{1}$, Artem Oprev ${ }^{1}$, Oleg Samodurov ${ }^{1}$, Artem Koptyuhov ${ }^{1}$, and Vladimir Ilatovskiy ${ }^{1}$ \\ ${ }^{1}$ NRC “Kurchatov Institute" - Petersburg Nuclear Physics Institute, Gatchina, Russia \\ ${ }^{2}$ P.L. Kapitza Institute for Physical Problems of the Russian Academy of Science, Production Department, Moscow, Russia
}

\begin{abstract}
The WWR-M reactor at PNPI is planned to be equipped with a high-flux source for ultracold neutrons (UCNs). The method of UCN production is based on neutron conversion in superfluid helium, exploiting the particular qualities of that quantum liquid. As a result of optimizing the source parameters, we expect a temperature of superfluid helium of $1.2 \mathrm{~K}$ and a UCN density of $1.3 \times 10^{4} \mathrm{~cm}^{-3}$ in a neutron electric dipole moment (EDM) spectrometer. The expected flux densities of cold neutrons (with wavelengths in the range 2-20 $\AA$ ) and very cold neutrons $(50-100 \AA)$ at the output of a neutron guide with a cross section of $30 \times 200 \mathrm{~mm}^{2}$ are $9.7 \times 10^{7} \mathrm{~cm}^{-2} \mathrm{~s}^{-1}$ and $8.3 \times 10^{5} \mathrm{~cm}^{-2} \mathrm{~s}^{-1}$, respectively. The capability of maintaining a temperature of $1.37 \mathrm{~K}$ at a thermal load of $60 \mathrm{~W}$ was shown experimentally, while the theoretical load is expected to be $37 \mathrm{~W}$. Calculations show that it is possible to decrease the helium temperature down to $1.2 \mathrm{~K}$ at similar heat load. The project includes the development of experimental stations at UCN beams, such as for a neutron EDM search, measurements of the neutron lifetime, and for a search for neutron-to-mirror-neutron transitions. In addition, three beams of cold and very cold neutrons are foreseen. At present, the vacuum container of the UCN source has been manufactured and the production of the low-temperature deuterium and helium parts of the source has been started.
\end{abstract}

\section{The project of a UCN source at the WWR-M reactor}

The project for creating $\mathrm{CN}$ and $\mathrm{UCN}$ sources originated in the $70 \mathrm{~s}$ of the last century. Efficient production of low energy neutrons at the WWR-M reactor complex employs cryogenic techniques. In early experiments, UCN fluxes high for that time were obtained here by means of passing reactor neutrons through various low-temperature converters: beryllium [1], liquid hydrogen [2], liquid deuterium [3], and solid deuterium [4]. In such converters, UCNs are formed by rare, single inelastic collisions where a neutron loses all its energy (in contrast to thermalization in a cold source, where reactor neutrons attain thermal equilibrium with a low-temperature medium with a corresponding shift of the whole spectrum to lower energies).

Yields of UCN production depend on the intensity of the reactor neutron flux and the efficiency of a cryogenic converter, with or without a cold premoderator. However, putting such a device close to the core of a reactor requires large radiation heats to be removed. This article describes technical solutions to the problem of maintaining a temperature as low as $1.2 \mathrm{~K}$ in a harsh radiation environment inside a reactor channel, which to our knowledge has not yet been accomplished anywhere else.

The WWR-M reactor offers a unique opportunity for a large suppression of thermal loads in a sufficiently

$\overline{\text { a e-mail: serebrov_ap@pnpi.nrcki.ru }}$ high thermal neutron flux in the order of $10^{12} \mathrm{~s}^{-1} \mathrm{~cm}^{-2}$. The preferred position for implementation is the so-called thermal column, a channel with a large diameter of $1 \mathrm{~m}$, adjoining to the reactor core [5,6], as shown in Fig. 1 (not to scale). This channel is large enough to place an assembly of graphite moderators $(23 \mathrm{~cm}$ thick) and $10 \mathrm{~cm}$ thick lead shields, a liquid deuterium premoderator kept at 20-22 K for cold neutrons, and, as central part, a converter of coldto-ultracold neutrons consisting of 35 litres of superfluid $\mathrm{He}(\mathrm{He}-\mathrm{II})$ to be held at $1.2 \mathrm{~K}$. The location allows us to reach a level of $37 \mathrm{~W}$ for the heat load on the superfluid He. A system of roots pumps existing at PNPI has sufficient pumping speed to remove this heat. Combined with cryogenic devices made by Linde, the whole cooling system is supposed to ensure stable operation of the UCN source at the WWR-M reactor.

Calculations of neutron fluxes and thermal loads have been made using MCNP programs [7]. Figure 1 indicates the heat in the various components generated by the reactor at $16 \mathrm{MW}$ power, notably, $15 \mathrm{~kW}$ in the lead shielding, $700 \mathrm{~W}$ in the liquid deuterium premoderator, $18 \mathrm{~W}$ in the aluminium shell of the central He chamber, and $19 \mathrm{~W}$ in the He-II. The thermal load on the source at $1.2 \mathrm{~K}$ temperature will thus be $37 \mathrm{~W}$.

Output optimization of UCN fluxes from the source has been carried out using a Monte-Carlo code [8], developed for neutron transport simulations with account of gravitation. It resulted in determining the geometry of the source chamber (diameter $30 \mathrm{~cm}$, volume $35 \mathrm{l}$ ) and the neutron guide from the UCN source. As a special feature, He vapours are pumped through the UCN guide, 


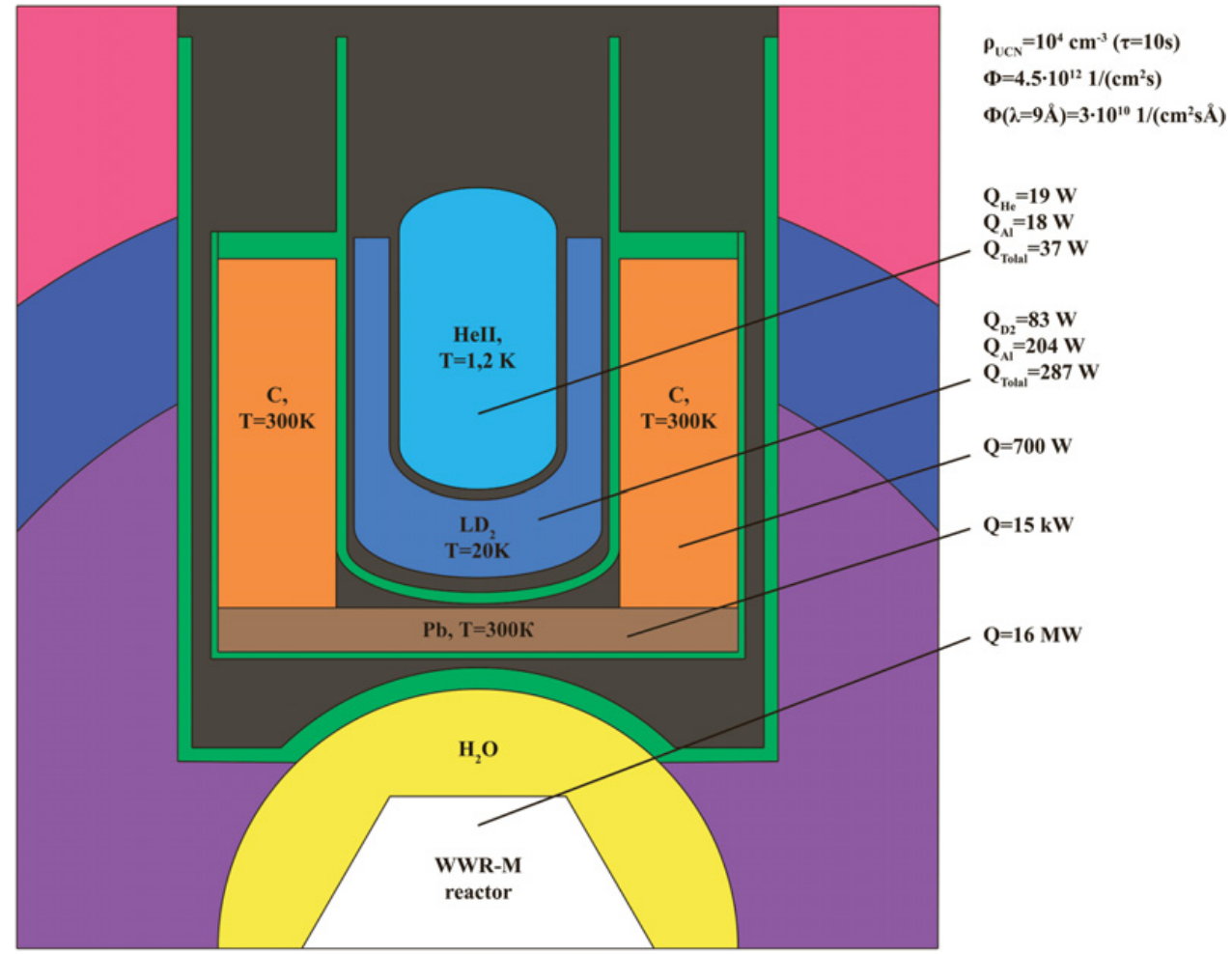

Figure 1. Location of the UCN source in the thermal column of the WWR-M reactor.

as the storage lifetime of neutrons in He gas at $15 \mathrm{~K}$ and a pressure of $50 \mathrm{~Pa}$ is still $52 \mathrm{~s}$, which is substantially longer than the time of UCN transport via the neutron guide. The chamber, neutron guides and external UCN traps representing UCN storage experiments connected to the source were simulated with a $58 \mathrm{NiMo}$ internal coating with velocity cut-off of $7.8 \mathrm{~m} / \mathrm{s}$ and $3 \times 10^{-4}$ loss factor.

Simulations provided theoretical UCN densities in external traps of 351 and 3501 . These sizes are typical for experiments to measure the electric dipole moment (EDM) of the neutron (351) [9], and the neutron lifetime (3501) [10]. Figure 2 shows the UCN density depending on the temperature of He-II in the source chamber. In addition, the axis of abscissae shows some values of neutron storage lifetimes in bulk He-II at the respective temperature. For comparison, Fig. 2 also gives UCN densities for a closed source chamber to show, what density could be achieved there in an accumulation mode. As we will rather work in a mode of stationary UCN production of the open source, the attainable density in the experimental trap of 351 is approximately 4 times lower. Note that for the trap with a volume by an order of magnitude larger it turns out to be only $54 \%$ less. That the density falls with increasing temperature is due to the decreasing neutron storage lifetime.

The new UCN source at the WWR-M reactor (Fig. 3) is supposed to improve the sensitivity to search for a neutron EDM (1) by two orders of magnitude, with large impact to search for physics beyond the standard model. Second, two installations for measuring the neutron lifetime are foreseen: one with a magnetic trap (2) and one with a big gravitational trap (4). Third, a setup to search of mirror dark matter (3) is planned. If the source will reach the projected production of $10^{8} \mathrm{UCN} / \mathrm{s}$, an experiment to search for neutron-antineutron oscillations based on the

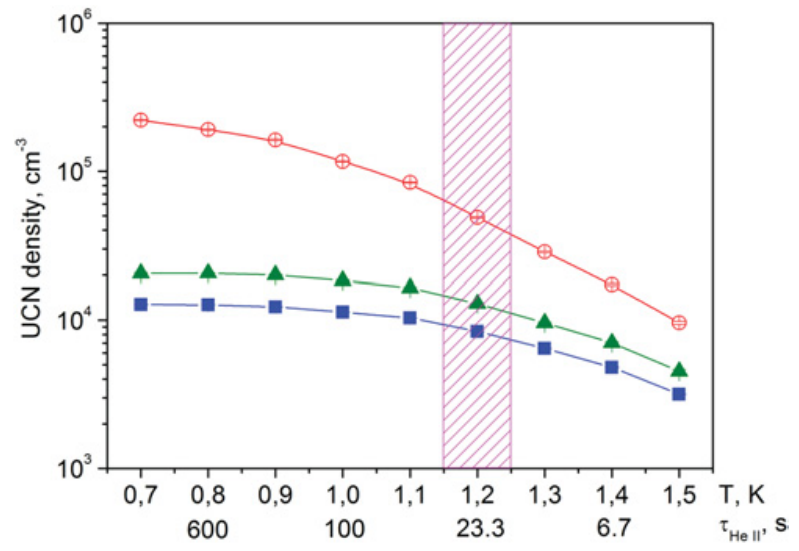

Figure 2. UCN density as a function of the He-II temperature - - in the closed source chamber, $\boldsymbol{\Lambda}$ - in a 35-1 external trap, in a 350-1 external trap.

storage of ultracold neutrons in a material trap will be proposed [11]. All installations have been developed and manufactured at PNPI and employed at UCN beams at the ILL in Grenoble, France. They are supposed to be transferred to PNPI once the new UCN source will become available. Enhancing the UCN intensity by more than two orders of magnitude will also enable to conduct principally new investigations.

A research program for condensed-matter physics on cold neutron beams is foreseen to be implemented at five experimental stations. Among those, four are ready for operation: a reflectometer (6), a polarimeter (7), a powder diffractometer (8) and a spin-echo spectrometer (9). Also foreseen is a beam of very cold neutrons (VCN) at $\mathrm{CN} 3$ for experiments to be defined in future. 


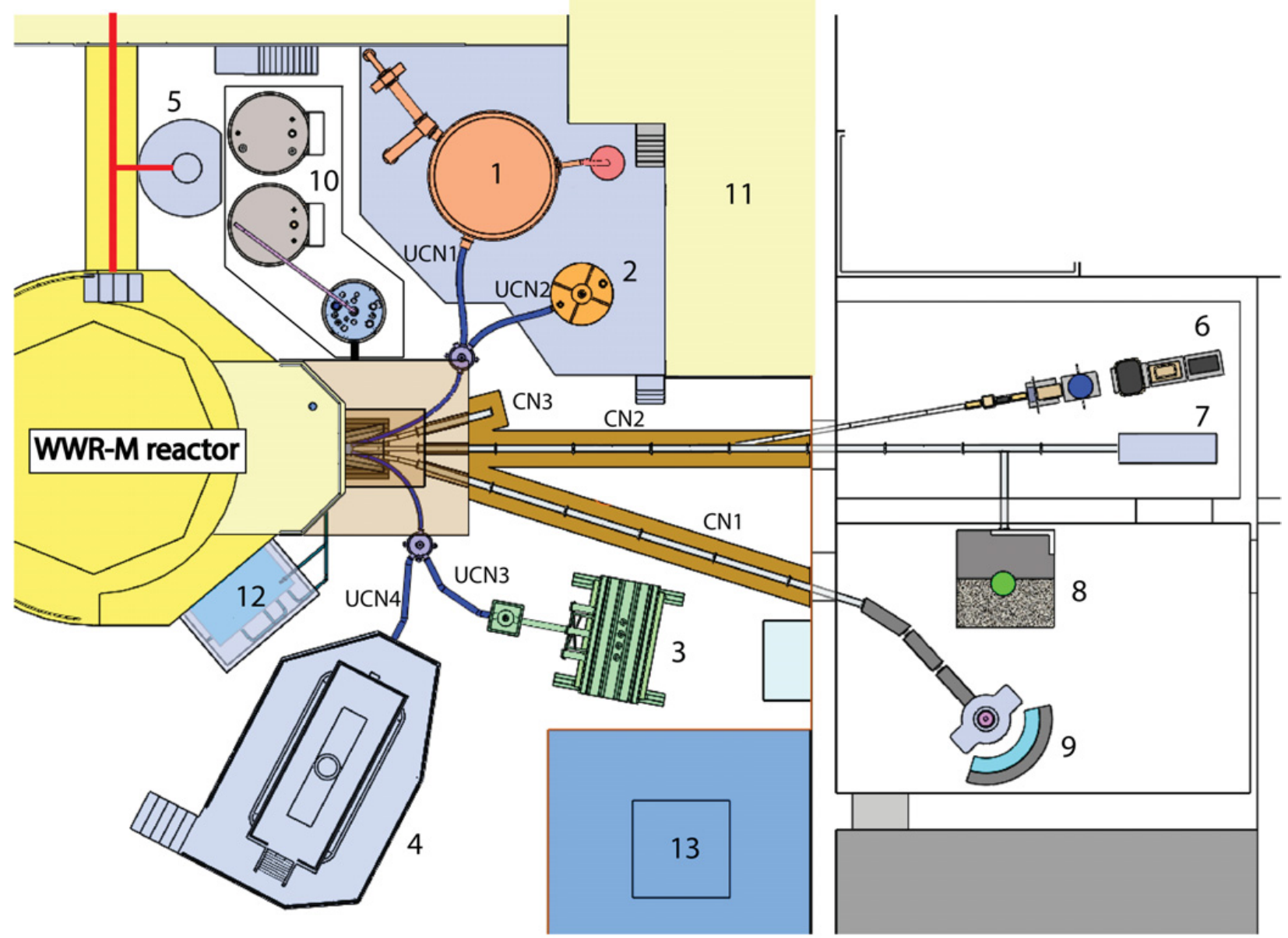

Figure 3. Schematic implementation of experimental equipment in the main hall of the WWR-M reactor hall. Beams of ultracold neutrons are named UCNx, cold and very cold neutron beams are named CNx. 1 - EDM spectrometer, 2 - UCN magnetic trap, 3 - n-n' experiment, 4 - UCN gravitational trap, 5 - Diffractometer, 6 - Reflectometer, 7 - Polarimeter, 8 - Powder diffractometer, 9 - Spin-echo spectrometer, $10-$ Cryogenic equipment for UCN source, $11-$ Technological platform for experimental equipment, 12 - Cooling system for the lead screen of the UCN source, 13 - Transport entrance to the main hall of the reactor.

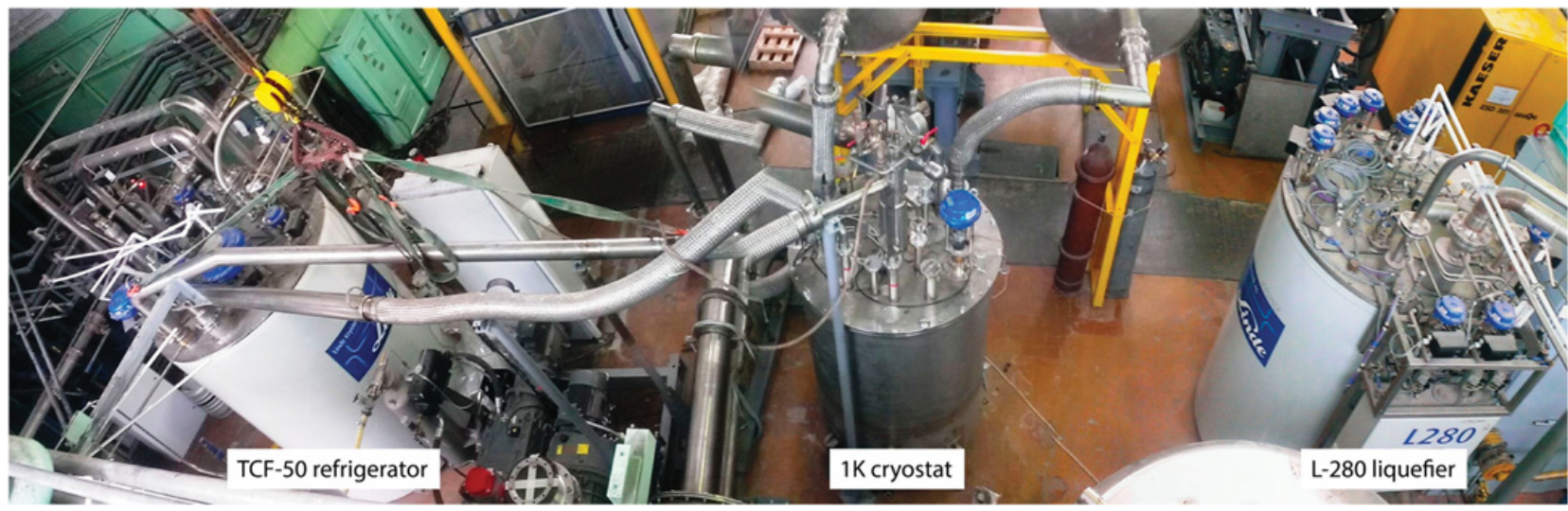

Figure 4. Photograph of the full-scale model of the UCN source for the WWR-M reactor.

\section{Full-scale tests of UCN source model}

For testing the project in practice, a full-scale model of the source was created (Fig. 4). Here, the thermalload-generating radiation was replaced by an electrical heater. Cooling cycles of the model were successfully launched 9 times. Detailed technical descriptions of the cryostat and the UCN model were already published in Ref. [12]. The model has allowed us to work out the technologies for generating and handling the superfluid and to demonstrate operation of the cryostat with $\mathrm{He}$ circulation, first still without added thermal loads. In a second step, measurements of the temperature dependence 


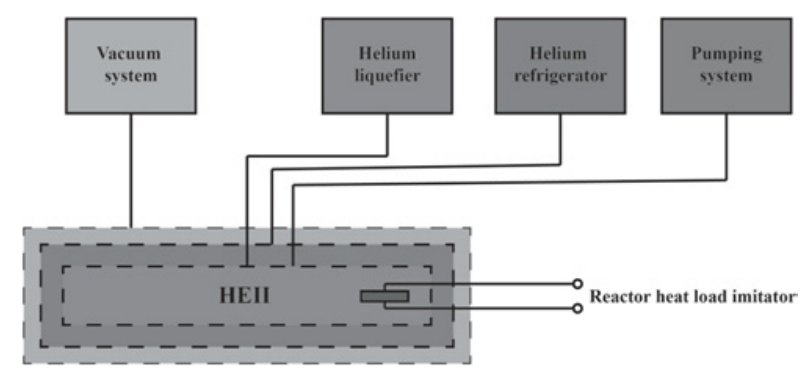

Figure 5. Schematic diagram of the technological equipment of the UCN source model.

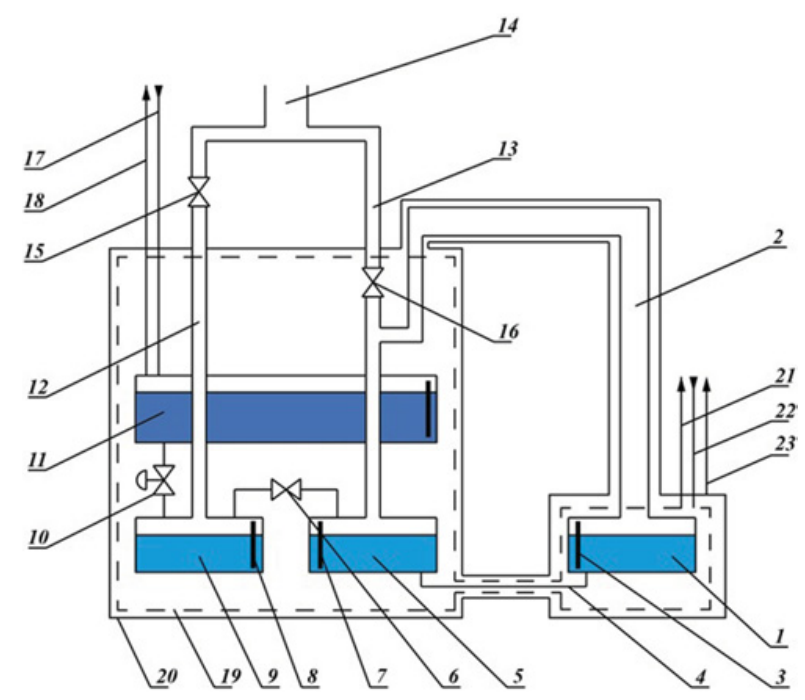

Figure 6. Schematic of the cryostat. See text for explanation of the numbered components.

Table 1. UCN source model electric power consumption.

\begin{tabular}{lc} 
Facility & Electric power consumption, $\mathbf{k W}$ \\
\hline L-280 liquefier & 160 \\
TCF-50 refrigerator & 315 \\
Pumping system & 95 \\
Water chiller & 95 \\
\hline
\end{tabular}

of the He-II bath were done with application of the thermal load by the heater.

A schematic diagram of the technological equipment of the UCN source model is shown in Fig. 5. Three major facilities in the complex maintain the helium at superfluid temperatures: a Linde L-280 liquefier, a Linde TCF-50 refrigerator, and an Edwards pumping system. The latter consists of three screw pumps (two GV600 and an EH4200) and four roots pumps (HV30000). This complex consumes over $665 \mathrm{~kW}$ electric power (see Table 1, where auxiliary systems such as air compressors and turbomolecular pumps were not included).

Figure 6 shows the schematic diagram of the lowtemperature parts of the UCN source. The chamber of the UCN source model (1) employed in our first tests has a volume of 351 . During operation, this chamber contains superfluid helium at $1.2 \mathrm{~K}$. A pipe (2) with a length of about $3 \mathrm{~m}$ is connected to the chamber and serves for pumping the evaporated helium. In addition, in the real source it will also serve as a neutron guide for UCN extraction. A pump-out pipeline (13) is connected

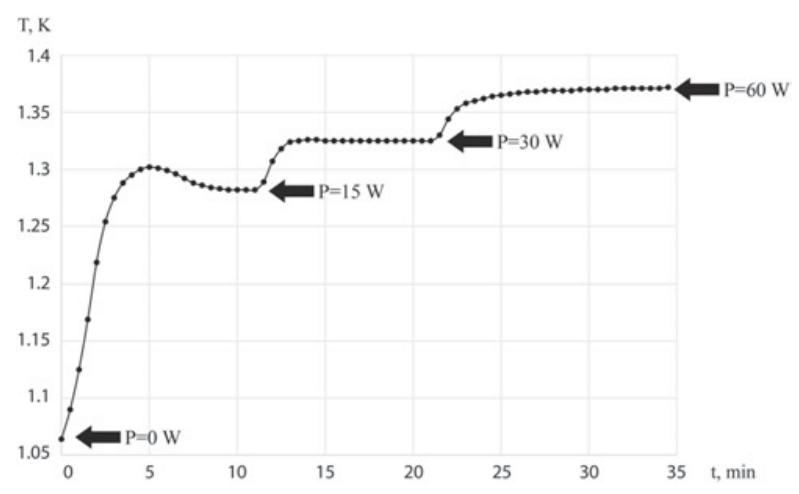

Figure 7. Experimental results for the full-scale model of UCN source.

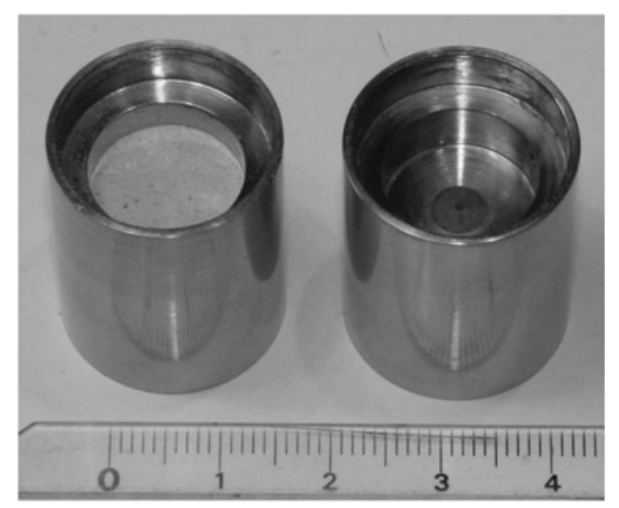

Figure 8. First prototypes of a superleak filter. They employ compressed $\mathrm{Al}_{2} \mathrm{O}_{3}$ powder with grain sizes $100 \mathrm{~nm}$ and $25 \mathrm{~nm}$, respectively.

vertically to the neutron guide (2). It removes helium vapours from the source model tank (1) and also from another lower tank (5). Liquid helium is supplied via a pipeline (17) from the helium liquefier to an upper tank (11) at $4.2 \mathrm{~K}$, which includes a level meter to monitor the liquid-helium content. Helium vapour from the tank (11) is returned to the liquefier via a pipe (18). Through a control valve (10), liquid helium is supplied to an intermediate tank (9) equipped with a level meter (8) and held at $1.2 \mathrm{~K}$ by pumping through a pipe (12). For thermal anchoring (to reduce heat influxes) the pump-out pipelines (12) and (13) pass through the tank (11), then are united into a common pipeline (14).

The superfluid-helium content in the lower tank (5) is monitored using a cryogenic valve (6) and a helium level gauge (7). These are used to fill/empty the lower tank (5) and the tank (1), where the helium level is also monitored (3). Both tanks contain helium at the same level and pressure, as they represent "communicating vessels" connected by a pipe (4). The pressure of helium vapour in the low-temperature part is maintained by the vacuum installation at a level of $55 \mathrm{~Pa}$, using pneumatic valves $(15,16)$.

The whole low-temperature part is surrounded by a heat screen (19) held at $15 \mathrm{~K}$ and is enclosed into a vacuum chamber (20). The heat screen (19) is cooled by circulating helium from the refrigerator via lines $(21,22)$. Another pumping line (23) serves for evacuation of the vacuum chamber by a turbomolecular pump.

Goals of the experiments with the heater were to demonstrate that we can maintain a sufficiently low 


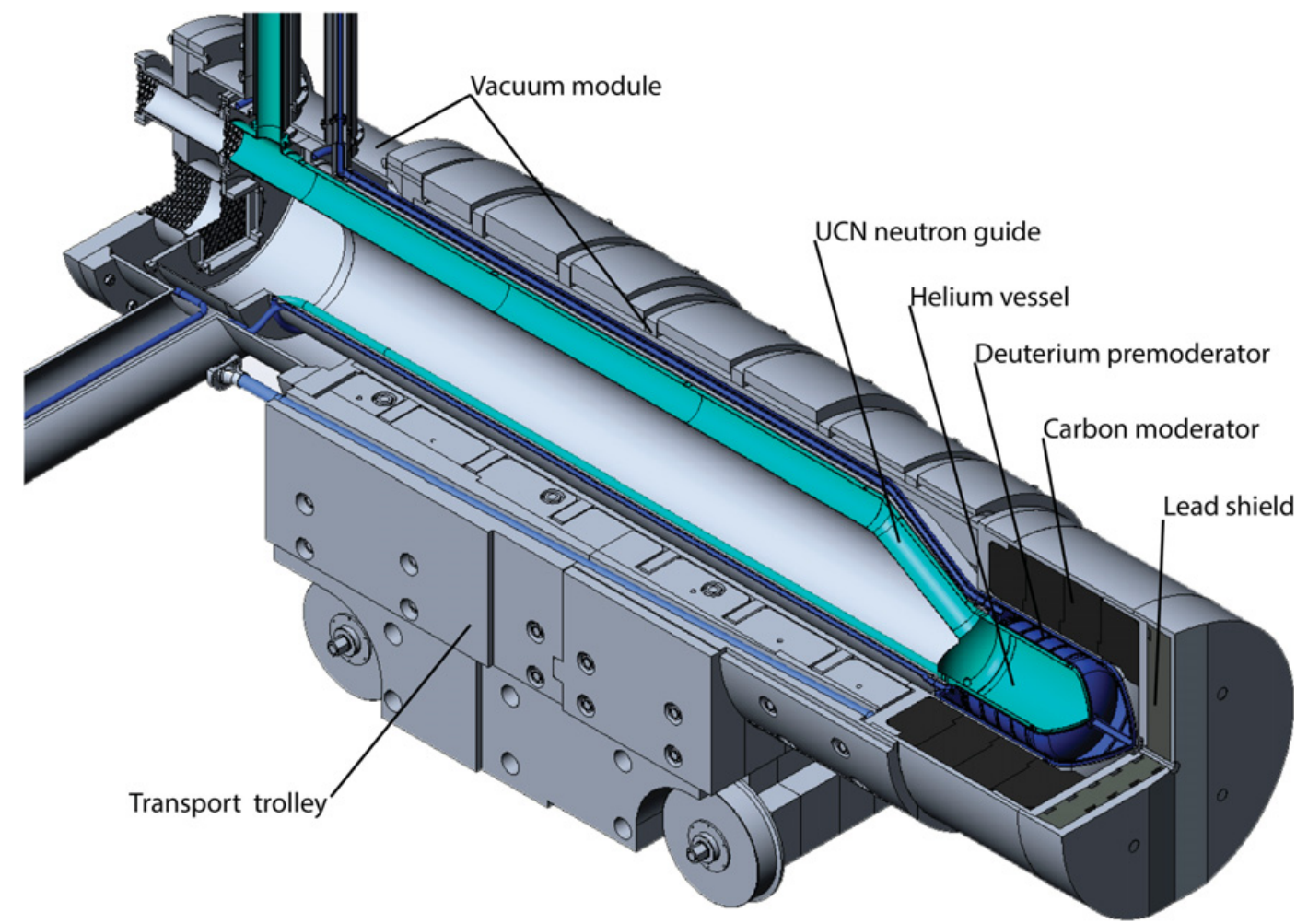

Figure 9. The UCN source assembled on its transport trolley.

He-II temperature, and to estimate the performance reserve of the pump system serving for circulation of the $\mathrm{He}$ gas. This estimation plays a crucial role for prediction of the source behaviour in the thermal column of the WWR-M reactor, since real heat loads are likely to be different from theoretical expectations. Experiments with the full-scale model measured temperatures of superfluid He at thermal loads up to $60 \mathrm{~W}$ (i.e., significantly higher than the estimated $37 \mathrm{~W}$ generated in the cold parts of the UCN source due to radiation from the reactor). In our experiments we used calibrated temperature sensors (delivered by Lake Shore Cryotronics, ensuring quality control according to ISO 9001:2008).

For a thermal load of $60 \mathrm{~W}$ the He-II temperature stabilised at $1.37 \mathrm{~K}$ (see Fig. 7). Despite so high loads on the UCN source model, the helium remains superfluid.

Analysis of the data has shown that the temperature of the superfluid He can still be substantially reduced. In the experiments, a part of the pumping line was passed though the cryostat (the branch behind the valve (16) in Fig. 6) in which its cross section was decreased to $50 \mathrm{~mm}$. We intend to redesign the pumping system so that the helium vapours will be pumped away bypassing the cryostat. That way we expect to obtain a temperature of $1.2 \mathrm{~K}$ at a thermal load of $40 \mathrm{~W}$.

The Linde L-280 liquefier is equipped with a purifier. It uses a coal absorber that is cooled down to $20 \mathrm{~K}$ by cold helium backflow from the upper bath (11) of the cryostat. The absorber removes impurities such as nitrogen, oxygen and even neon and hydrogen. During cooldown and regeneration of the absorber the performance of the liquefier strongly decreases (even stops), which we need to take into account in the whole cooling cycle. Nevertheless, the liquefier performance is still sufficient for cleaning and liquefying the required quantity of $\mathrm{He}$ during preparation of the upper bath. An uninterrupted operation of the technological complex can thus be ensured.

The UCN source will finally be equipped with a superleak filter, for removal of the strongly neutronabsorbing ${ }^{3} \mathrm{He}$ (see p. 1233 in Ref. [6]). The filter will replace the valve (6) between the tanks (5) and (9). For the experiments described here it was not needed and accordingly not yet included in the system.

A first prototype of a superleak filter was already tested (Fig. 8). It showed $2.5 \mathrm{~g} / \mathrm{s}$ performance at $1.2 \mathrm{~K}$. At temperature in the range $2.3-2.4 \mathrm{~K}$, the average helium flow rate through superleak filter was $4.3 \times 10^{-4} \mathrm{~g} / \mathrm{s}$. With a fraction of $\sim 10^{-6}$ of ${ }^{3} \mathrm{He}$ in natural helium, the penetration of ${ }^{3} \mathrm{He}$ through the filter does not exceed $4 \times 10^{-10} \mathrm{~g} / \mathrm{s}$. Recently, we manufactured a superleak filter with $3 \mathrm{~g} / \mathrm{s}$ performance. It will soon be tested at the UCN source model.

Concluding this experimental section we note that the presented work carried out on a full scale model provides an experimental justification for installing the projected UCN source at the WWR-M reactor.

\section{Brief descriptions of the source components}

The UCN source for the reactor WWR-M is made of three units (Fig. 9) described in subsequent sections:

1. Vacuum module,

2. Cryogenic module,

3. Transport trolley.

The source is manufactured in the workshop of NRC "Kurchatov Institute" at PNPI. At present, the vacuum module and the transport trolley have been completed, as well as separate units of the cryogenic module. 


\subsection{Vacuum module}

The vacuum module complies with safety standards to ensure structural stability of the outer shell in case of a hypothetical emergency explosion inside the module due to ignition of a hydrogen-air mixture of stoichiometric composition. It has a water-cooled lead shield designed for reducing the heat load due to gamma quanta on lowtemperature parts of the UCN source. At full reactor power, the thermal load on the shield is $15 \mathrm{~kW}$. 64 graphite blocks installed still within the vacuum module thermalize neutrons from the reactor.

\subsection{Cryogenic module}

The cryogenic module consists of a deuterium and a helium part. The former includes a vessel of 50 litres for the liquid-deuterium premoderator. Deuterium gas is liquefied with a blanket of gaseous helium at $20 \mathrm{~K}$. The low helium temperature is maintained by a refrigerator providing $3 \mathrm{~kW}$ power at $15 \mathrm{~K}$. The unit is equipped with a multi-step security system for prevention of the formation of hydrogen-oxygen mixtures.

The central part of the cryogenic module is the chamber for 35 litres of superfluid He. It is connected to a pumping line via a neutron guide of $100 \mathrm{~mm}$ diameter. The mass flow of helium through the source chamber is $1.5 \mathrm{~g} / \mathrm{s}$.

\subsection{Transport trolley}

The transport trolley is designed for moving UCN source elements into and out of the thermal column of the reactor.
Its volume exactly fills the thermal column for creation of an internal biological protection. To reduce activation, the trolley and shielding units are made of aluminium alloys. The total weight of the trolley is 10 tons.

This research has been performed at NRC "Kurchatov Institute" - PNPI and is supported by RSF (project No. 14-22-00105).

\section{References}

[1] I.S. Altarev et al., Preprint PNPI-246 (1976)

[2] I.S. Altarev et al., Phys. Lett. A 80, 413 (1980)

[3] I.S. Altarev et al., JETP Lett. 44, 269 (1986)

[4] A.P. Serebrov et al., JETP Lett. 62, 764 (1995)

[5] A.P. Serebrov et al., Vestnik of Saint Petersburg University 2, 27 (2015)

[6] A.P. Serebrov, Crystallography Reports 56, 1230 (2011)

[7] J.F. Briesmeister, MCNP - A General Monte Carlo $N$-Particle Transport Code, Version 4C (2000)

[8] A.K. Fomin, Modeling of experiments with ultracold neutrons. PhD Thesis (2006)

[9] A.P. Serebrov et al., JETP Lett. 99, 4 (2014)

[10] A.P. Serebrov et al., Phys. Rev. C 97, 055503 (2018)

[11] A.K. Fomin et al., The 3rd International Conference on Particle Physics and Astrophysics, KnE Energy \& Physics, 109 (2018)

[12] A.P. Serebrov et al., Tech. Phys. Russian J. Appl. Phys. 62, 329 (2017) 